Method The clinical and investigation findings in a series of adult patients with SMART syndrome presenting primarily with seizures were reviewed.

Results Four patients with SMART syndrome presenting with seizures were identified (mean age 51 years). Mean time from radiation therapy to SMART syndrome was 22.5 years (range 15-32 years). Indications for radiation were primary brain tumour (three patients) and haematological malignancy (one patient). Two patients had a history of seizures prior to SMART syndrome. Three patients had headaches at presentation. All patients presented with focal impaired awareness seizures with motor features. One patient had refractory nonconvulsive status epilepticus requiring intravenous anaesthesia. Three patients had persistent negative motor deficits, associated with ongoing electrographic seizures with no clinical correlate, only identified on repeated EEGs or continuous EEG (cEEG). All patients failed initial anti-seizure medications (ASM), requiring a mean of five ASMs for seizure control. All patients had enhancing cortical MRI changes consistent with SMART syndrome that corresponded to the clinical deficit and ictal changes on EEG. At follow-up all patients improved but had persistent neurological deficits.

Conclusion SMART syndrome presents with seizures and less frequently status epilepticus and may be the basis for the associated clinical features and radiologic abnormalities. Judicious use of EEG and where necessary CEEG to identify non-convulsive seizures should be considered in patients with SMART syndrome presenting with prolonged neurological deficits.

\section{OPTIC PERINEURITIS IN GIANT CELL ARTERITIS}

1,2Elaine Pang, 1,2,3,4 Neha Irani. 'Fiona Stanley Hospital, Murdoch, WA, Australia; ${ }^{2}$ Sir Charles Gairdner Hospital, Nedlands, WA, Australia; ${ }^{3}$ Fremantle Hospital, Fremantle, WA, Australia; ${ }^{4}$ Joondalup Health Campus, Perth, WA, Australia

\subsection{6/bmino-2021-ANZAN.99}

Background Optic perineuritis (OPN) is a rare orbital inflammatory disease which targets the optic nerve sheath. Although OPN is predominantly idiopathic, it can be secondary to an array of inflammatory, infective or malignant conditions, including giant cell arteritis (GCA).

Methods/Results We describe a case of a 75 -year-old man with a four-week history of headaches with associated periorbital swelling, and monocular decrease visual acuity without significant constitutional or systemic symptoms. This was in the context of initially normal ESR and CRP. MRI head demonstrated bilateral OPN and GCA was subsequently confirmed based on temporal artery biopsy. He was managed with high dose prednisolone and upadacitinib.

Conclusions This case highlights the perineuritis as a rare manifestation of GCA.

\section{DEVELOPING A QUALITY ASSURANCE FRAMEWORK FOR NEURO-OPHTHALMOLOGY REFERRALS USING NODE - THE NEURO-OPHTHALMOLOGY DATABASE}

${ }^{1}$ Anoushka Lal, ${ }^{1}$ Olga Roche, ${ }^{1}$ Owen White, ${ }^{1}$ Wendy Wang, ${ }^{2}$ Shivanand Sheth, ${ }^{2}$ Rahul Chakrabarti, ${ }^{2}$ Lana del Porto, ${ }^{2}$ Anthony Fok, ${ }^{2}$ Neil Shuey, ${ }^{3}$ Clare Fraser, ${ }^{2}$ Lionel Kowal, ${ }^{1}$ Anneke Van der Walt. 'Department of Neuroscience, Central Clinical School, Monash University, Melbourne, VIC, Australia; ${ }^{2}$ Royal Victorian Eye and Ear Hospital, Melbourne, VIC, Australia; ${ }^{3}$ Sydney Eye Hospital, Sydney, NSW, Australia

10.1136/bmjno-2021-ANZAN.100
Objective Quality assurance (QA) in neuro-ophthalmology $(\mathrm{NOPH})$ is often lacking. The QA registry, NODE (Neuroophthalmology Database), was established and implemented in tertiary NOPH clinics in Australia. We developed a consensus on triage categories according to Australian standardised triage categories;P1 (consult $<=30$ days), P2 (consult $<=30-60$ days) and P3 (consult $>60$ days).

Methods Data on 410 patients at Alfred Hospital, Melbourne was collected with NODE. We developed a consensus on assignation of NOPH conditions to triage categories using recommendations from a panel of neuro-ophthalmologists with the modified Delphi approach. The average days from referral to triage and triage to the initial consultation were compared to the developed triage category standard.

Results Most patients presenting to the service were female $(n=262,64 \%)$, aged 21 to 30 years. Common diagnoses were Idiopathic Intracranial Hypertension, IIH (24\%), Optic Neuropathy, ON (17\%), Headaches, (11\%) Cranial Nerve Defects, CND (9\%) and Eye Movement Disorders, EOMD (9\%). The mean time from referral to triage was $<2$ days for all the common NOPH conditions. The mean time (days, +-standard deviation) from P1 category triage to initial consult for IIH was $26( \pm 7)$, ON $27( \pm 11)$, and CND was $17( \pm 5)$. The mean time (days) from P2 triage to initial consultant for Headaches was $27( \pm 12)$, and EOMD was $( \pm 17)$. The mean time (days) from P3 triage to initial consultant for Myasthenia Gravis was $30( \pm 10)$.

Conclusion We have established a consensus agreement on triage categories for neuro-ophthalmological conditions. We established a QA framework for other NOPH clinics in Australia.

\section{EMBOUCHURE DYSTONIA COMPLICATING A CASE OF FOCAL CEREBRAL VASCULITIS}

'Ariadna Fontes-Villalba, ${ }^{2}$ Suran Fernando, 'John Parratt. 'Neurology, Royal North Shore Hospital, Sydney, NSW, Australia; ${ }^{2}$ Department of Immunology, Royal North Shore Hospital, Sydney, NSW, Australia

\subsection{6/bmjno-2021-ANZAN.101}

Background Primary central nervous system vasculitis (PCNSV) is a rare disorder that normally develops with bilateral brain lesions. We report a case of pathologically confirmed hemispheric PCNSV with associated brain atrophy and symptoms of focal dystonia.

Method Case report.

Result A 45-year-old female presented with fluctuating apraxia, dystonia, cognitive dysfunction and numbness of the face. She had embouchure dystonia. She was taking lamotrigine for a longstanding history of well controlled partial seizures. A cerebral MRI revealed multiple left hemispheric cortical and subcortical white matter lesions. Many of the lesions enhanced. The lesions came and went over a period of three years but cortical atrophy of the fronto-parietal gyri developed. The CSF demonstrated matched oligoclonal bands. The autoimmune serology was negative. A cerebral PET demonstrated hypo-metabolism in the affected areas.A cortical lesion that was enhancing was biopsied and the histology showed several small foci of punctuate necrosis (infarcts) with lymphohistiocytic infiltration of blood vessels consistent with small vessel vasculitis. The patient was treated with prednisone and mycophenolate and improved clinically, albeit her clarinet playing 
did not return to pre-morbid levels. The MRI lesions resolved and did not recur.

Conclusion Focal vasculitis is rare but may result in neuronal loss and specific cortical damage and atrophy, in this case leading to embouchure dystonia.

\section{REFERENCES}

1. Salvarani C, Brown RD Jr, Hunder GG. Adult primary central nervous system vasculitis. Lancet 2012 Aug 25;380(9843):767-77.

2. Ho MG, Chai W, Vinters HV, Hathout G, Mishra S, Yim C, Valdes-Sueiras M, Nishimura R. Unilateral hemispheric primary angiitis of the central nervous system. J Neurol 2011 Sep;258(9):1714-6.

3. Unihemispheric central nervous syste.

\section{LAMBERT EATON MYAESTHENIC SYNDROME IN THE ABSENCE OF MALIGNANCY}

Joseph B Stockwell, Leon S Edwards, Daniel Wardman, Neil Griffith. Neurology, Liverpool Hospital, Sydney, NSW, Australia

\subsection{6/bmjno-2021-ANZAN.102}

Introduction We report a case of Lambert Eaton Myaesthenic Syndrome (LEMS) in an 85-year-old gentleman with no active malignancy.

Case An 85-year-old gentleman presented with a 3-month history of proximal weakness, confusion, nausea and vomiting. His medical history included gastric adenocarcinoma with curative resection 21-years ago and a 2-year history of a stable sensorimotor peripheral neuropathy. During his admission he experienced an episode of new onset fluctuating diplopia. Neurological examination demonstrated mild upper and lower limb non-fatiguable weakness. There was no detectable cranial nerve palsy.

A myasthenia antibody panel was ordered. Voltage-gatedcalcium-channel-antibodies were positive $(47 \mathrm{pM})$ (normal range $<30 \mathrm{pM}$ ). Repetitive nerve stimulation demonstrated an increment in compound muscle action potential of the right nasalis and right abductor digiti minimi following exercise and highrate stimulation consistent with the clinical diagnosis of LEMS. Investigation for malignancy including tumour markers, CT chest, abdomen and pelvis, MRI-pancreas and whole body PET scan were unremarkable.

The patient underwent a 1-month period of inpatient rehabilitation and was discharged home. At 6 months, he remains well with no further episodes of diplopia or weakness. To date, no malignancy has been identified.

Conclusion LEMS in absence of an identified malignancy is an uncommon diagnosis. Those cases that have been documented are also more likely to occur in younger patients. The case we present here highlights a constellation of vague seemingly discordant symptoms with a unifying diagnosis and offers the patient a chance to be actively monitored for the development of malignancy in the future.

\section{AN UNDIFFERENTIATED AUTOIMMUNE NEUROINFLAMMATORY ILLNESS ASSOCIATED WITH LOW CSF HYPOCRETIN \& CENTRAL HYPOTHALAMIC DYSREGULATION}

${ }^{1}$ Hoang-Mai Dinh, ${ }^{1}$ Nicholas Gazy, 1,2 Logan Gardner, 'Stephen Tisch, ${ }^{1}$ Andrew Carr. ${ }^{1} S t$ Vincent's Hospital Sydney, Sydney, NSW, Australia; '2Westmead Hospital, Sydney, NSW, Australia

10.1136/bmjno-2021-ANZAN.103
Introduction This case report explores a possible undifferentiated autoimmune neuroinflammatory illness presenting with recurrent fevers, abdominal pain, hypersomnolence and sleep attacks with low cerebrospinal fluid (CSF) hypocretin, and a partial response to anakinra, a human interleukin 1 receptor antagonist.

Case Presentation A 19 year old female presented with 5 years of abdominal pain and fatigue with no clear aetiology identified following extensive investigation. She subsequently was found to have recurrent fevers to $38^{\circ} \mathrm{C}$, an intermittent fine, macular rash and sudden sleeping at inappropriate times. Her brain MRI was normal and (CSF) showed normal protein and no white cells, but a low hypocretin level ( $<200$ units). Further investigations including whole exome sequencing, gastrointestinal, autoimmune and metabolic assessments, yielded limited findings. Previous therapy with colchicine had been ineffective.

Management and Outcome For a presumptive diagnosis of an undifferentiated autoinflammatory disorder, she was received prednisolone $10 \mathrm{mg}$ daily for 4 weeks with no benefit. She then initiated anakinra, which improved in rash and sleep attacks. Despite initially controlling her recurrent fevers for a period of four weeks, this symptom ultimately recurred, with ongoing abdominal pain.

Discussion Low levels of hypocretin in the CSF has been associated with narcolepsy type 1 and has thought to been associated with an undefined autoimmune mechanism. It is hypothesised that her hypothalamic orexin has been altered due to these inflammatory changes leading to body temperature dysregulation and sleep disorder. Interestingly the hypersomnolence appear to have improved with anakinra, a therapy not typically used in narcolepsy.

\section{OVERLAPPING AUTOIMMUNITY: A CASE OF CONCOMITANT AQUAPORIN-4 AND MYELIN OLIGODENDROCYTE GLYCOPROTEIN (MOG) ANTIBODY POSITIVITY IN NEUROMYELITIS OPTICA SPECTRUM DISORDER}

\footnotetext{
${ }^{1,2}$ Miriam Wronski, ${ }^{2}$ Justine J Wang. ${ }^{1}$ Royal North Shore Hospital, Sydney, NSW, Australia; ${ }^{2}$ St George Hospital, Sydney, NSW, Australia
}

10.1136/bmjno-2021-ANZAN.104

Objectives To describe a rare case of double antibody positive Neuromyelitis Optica Spectrum Disorder (NMOSD) with both Aquaporin-4 and MOG antibodies, occurring following a Pertussis infection in a patient with a history of auto-immunity.

Methods Retrospective review of clinical records.

Results A 41-year-old Chinese woman with a history of Systemic Lupus Erythematosus presented with a sub-acute onset of progressive gait ataxia and urinary retention occurring seven days after a confirmed Bordetella pertussis infection. Magnetic resonance imaging revealed extensive subcortical and thalamic T2/FLAIR hyperintensities with subtle enhancement, and a longitudinally extensive non-enhancing spinal cord lesion (T1-T7), without optic nerve involvement. Cerebrospinal fluid protein was raised $(0.55 \mathrm{~g} / \mathrm{L})$ with 7 mononuclear cells and matched oligoclonal bands. Viral PCRs were negative including JC virus and Pertussis. Established live cell-based immunoassays revealed positivity for both Aquaporin-4 antibodies (in CSF and serum) and MOG antibodies in high 